Stefano Ferrari, Pietro Ruggieri, Emanuela Palmerini, Marco Alberghini, Piero Picci, and Gaetano Bacci, Istituto Ortopedico Rizzoli, Bologna; Graziella Cefalo and Rossella Bertulli, Istituto Nazionale Tumori; Antonina Parafioriti, Istituto Gaetano Pini, Milano; Angela Tamburini, Ospedale Meyer; Rodolfo Capanna,

Azienda Ospedaliera Universitaria

Careggi, Firenze; Franca Fagioli and Alessandra Linari, Ospedale Infantile Regina Margherita; Alessandro Comandone,

Ospedale Gradenigo, Torino; and Gianni Bisogno, Oncologia Pediatrica, Università, Padova, Italy.

Submitted July 21, 2011; accepted March 6, 2012; published online ahead of print at www.jco.org on May 7 , 2012.

Supported by the Italian Ministry of Health-Project Alleanza Contro il Cancro and the Italian Sarcoma Group Research Funds.

Authors' disclosures of potential conflicts of interest and author contributions are found at the end of this article.

Clinical Trials repository link available on JCO.org.

Corresponding author: Stefano Ferrari, MD, Istituto Ortopedico Rizzoli, Via Pupilli 1, 40136 Bologna, Italy; e-mail: stefano.ferrari@ior.it.

() 2012 by American Society of Clinical Oncology

0732-183X/12/3017-2112/\$20.00 DOI: $10.1200 / J C O .2011 .38 .4420$

\title{
Neoadjuvant Chemotherapy With Methotrexate, Cisplatin, and Doxorubicin With or Without Ifosfamide in Nonmetastatic Osteosarcoma of the Extremity: An Italian Sarcoma Group Trial ISG/OS-1
}

Stefano Ferrari, Pietro Ruggieri, Graziella Cefalo, Angela Tamburini, Rodolfo Capanna, Franca Fagioli, Alessandro Comandone, Rossella Bertulli, Gianni Bisogno, Emanuela Palmerini, Marco Alberghini, Antonina Parafioriti, Alessandra Linari, Piero Picci, and Gaetano Bacci

See accompanying editorial on page 2033

$$
\begin{array}{llllllll}
\text { A } & \text { B } & \text { S } & \text { T } & \text { R } & \text { A } & \text { C } & \text { T }
\end{array}
$$

\section{Purpose}

We compared two chemotherapy regimens that included methotrexate (MTX), cisplatin (CDP), and doxorubicin (ADM) with or without ifosfamide (IFO) in patients with nonmetastatic osteosarcoma of the extremity.

\section{Patients and Methods}

Patients age $\leq 40$ years randomly received regimens with the same cumulative doses of drugs (ADM $420 \mathrm{mg} / \mathrm{m}^{2}$, MTX $120 \mathrm{~g} / \mathrm{m}^{2}$, CDP $600 \mathrm{mg} / \mathrm{m}^{2}$, and IFO $30 \mathrm{~g} / \mathrm{m}^{2}$ ) but with different durations (arm A, 44 weeks; arm B, 34 weeks). IFO was given postoperatively when pathologic response to MTX-CDP-ADM was poor (arm A) or given in the primary phase of chemotherapy with MTX-CDPADM (arm B). End points of the study included pathologic response to preoperative chemotherapy, toxicity, and survival. Given the feasibility of accrual, the statistical plan only permitted detection of a $15 \%$ difference in 5 -year overall survival (OS).

\section{Results}

From April 2001 to December 2006, 246 patients were enrolled. Two hundred thirty patients (94\%) underwent limb salvage surgery (arm A, 92\%; arm B, 96\%; $P=.5$ ). Chemotherapy-induced necrosis was good in $45 \%$ of patients $(48 \%$ in $\operatorname{arm~} A, 42 \%$ in $\operatorname{arm} B ; P=.3)$. Four patients died of treatment-related toxicity ( $\operatorname{arm} A, n=1 ; \operatorname{arm} B, n=3)$. A significantly higher incidence of hematologic toxicity was reported in arm B. With a median follow-up of 66 months (range, 1 to 104 months), 5-year OS and event-free survival (EFS) rates were not significantly different between $\operatorname{arm} \mathrm{A}$ and $\operatorname{arm} \mathrm{B}$, with OS being $73 \%(95 \% \mathrm{Cl}, 65 \%$ to $81 \%)$ in arm $\mathrm{A}$ and $74 \%(95 \% \mathrm{Cl}, 66 \%$ to $82 \%)$ in arm B and EFS being $64 \%(95 \% \mathrm{Cl}, 56 \%$ to $73 \%)$ in $\operatorname{arm~} \mathrm{A}$ and $55 \%(95 \% \mathrm{Cl}, 46 \%$ to $64 \%$ ) in arm B.

\section{Conclusion}

IFO added to MTX, CDP, and ADM from the preoperative phase does not improve the good responder rate and increases hematologic toxicity. IFO should only be considered in patients who have a poor histologic response to MTX, CDP, and ADM.

\section{J Clin Oncol 30:2112-2118. (C) 2012 by American Society of Clinical Oncology}

\section{INTRODUCTION}

Probability of survival in patients with nonmetastatic osteosarcoma of the extremity has improved dramatically with the addition of chemotherapy tosurgicalremovalofthetumor. ${ }^{1}$ Multiagentchemotherapy has been used since the 1970s, but a standard chemotherapy regimen has not yet been defined. $^{2}$

A previous clinical trial conducted by the Italian Sarcoma Group (ISG) and the Scandinavian Sar- coma Group (SSG), ISG/SSG-1, used the following four active drugs against osteosarcoma: methotrexate (MTX), cisplatin (CDP), doxorubicin (ADM), and ifosfamide (IFO). ${ }^{3}$ ISG/SSG-1 involved an intensified chemotherapy treatment that included IFO delivered at a high dose $\left(3 \mathrm{~g} / \mathrm{m}^{2}\right.$ per day over a 5-day continuous infusion). ${ }^{3}$ Results of the study showed that addition of high-dose IFO to MTX, $\mathrm{CDP}$, and $\mathrm{ADM}$ was feasible, but survival rates were similar to those obtained with less intensive fourdrug regimens using standard-dose IFO. ${ }^{3}$ 
A new study (ISG/OS-1) was subsequently designed by the ISG with the aim of optimizing the drug administration schedule. Two different chemotherapy regimens were compared, both based on the four active drugs given according to different schemes, but with the same cumulative doses. Protocols differed in terms of dose-intensity and modality of use of IFO. Final results of the ISG/OS-1 study are reported here.

\section{PATIENTS AND METHODS}

\section{Study Design}

ISG/OS-1 was a multicenter randomized trial comparing efficacy and toxicity of two chemotherapy regimens, both based on MTX, CDP, ADM, and IFO and with the same cumulative doses but administered according to different schemes. The protocols had a different dose-intensity and different modality of use of IFO. In arm A, IFO was given only postoperatively and in patients with poor histologic response to primary chemotherapy with MTX, $\mathrm{CDP}$, and ADM. In arm B, IFO was delivered in all patients and in the primary phase of chemotherapy.

\section{Random Assignment}

The Istituto Ortopedico Rizzoli Ethic Committee secretariat was responsible for the random assignment procedures. For allocation of the participant patients, a computer-generated list of random numbers was used. Eligible patients were randomly assigned by fax to arm A or B.

\section{Patient Selection}

Eligibility criteria for entry onto the study were diagnosis of primary, central, high-grade osteosarcoma of the extremity; age $\leq 40$ years; and normal hepatic, renal, bone marrow, and cardiac function. The local ethics committees approved the protocol. After explanation of the research study and of the related procedures, a written informed consent was obtained from the adult patients or from legally authorized representatives if the patients were minors.

Representative histologic slides and radiographic images were centrally reviewed. Exclusion criteria were as follows: evidence of overt metastatic disease at diagnosis, previous treatment for osteosarcoma, medical contraindications to the drugs included in the protocol, and lack of availability for follow-up.

The primary tumor was evaluated on plain radiographs, computed tomography (CT) scans, and/or magnetic resonance imaging scans. Screening for metastases was by bone scintigraphy and CT scanning of the chest. Baseline studies included CBC, serum electrolytes, and glomerular filtration rate estimation; serum alkaline phosphatase and lactate dehydrogenase levels; bilirubin and aminotransferase levels; and echocardiography.

\section{Treatment Plan and Follow-Up}

Eligible patients underwent primary chemotherapy. Before surgery, CT and/or magnetic resonance imaging of the tumor and CT of the chest were repeated. After surgery, surgical margins were assessed according to Enneking et $\mathrm{al},{ }^{4}$ and histologic analysis of the tumor map was performed in accordance with a method reported previously. ${ }^{5}$ When the percentage of tumor necrosis was $\geq 90 \%$, patients were classified as good responders (GRs); when the percentage of tumor necrosis was lower, patients were defined poor responders (PRs). Diagnosis, histologic subtype, and histologic response were reviewed by an expert panel of pathologists. After completion of chemotherapy, patients were observed by $\mathrm{x}$-ray of the operated limb and CT scan of the chest every 3 months for 2 years, at 4-month intervals during the third and fourth years, and subsequently every 6 months.

\section{Chemotherapy}

The CONSORT diagram is in Figure 1, and the chemotherapy protocol is outlined in Figure 2. Cumulative doses (MTX $120 \mathrm{~g} / \mathrm{m}^{2}$, CDP $600 \mathrm{mg} / \mathrm{m}^{2}$, $\mathrm{ADM} 420 \mathrm{mg} / \mathrm{m}^{2}$, and IFO $30 \mathrm{~g} / \mathrm{m}^{2}$ ) were the same in the two arms. The planned overall duration of treatment was 43 weeks for arm A ( 34 weeks in case of good histologic response) and 34 weeks for arm B. Details on chemotherapy administration are listed in Appendix Table A1 (online only).

\section{Statistics}

A total of 246 patients was required to detect a survival difference of $15 \%$ or higher at 5 years between the two groups (study power of $80 \%$, type I error of $5 \%$ ). With an expected enrollment rate of 45 to 50 patients per year, the recruitment period was set at 5 years.

The two arms were compared in terms of protocol compliance, toxicity, and survival. Compliance with the protocol was evaluated for each patient and was expressed in terms of relative received dose of each drug (cumulative received dose to cumulative protocol-planned dose [in milligrams per square meter] ratio). Furthermore, for each patient, the received dose-intensity (RD/I) was calculated by dividing the average of relative received cumulative doses by the relative chemotherapy duration (ratio between actual duration of chemotherapy treatment and protocol-planned duration). The $t$ test was used for comparing protocol compliance between the two regimens.

Toxicity was graded according to the National Cancer Institute Common Toxicity Criteria (version 2.0). Toxicity was assessed by comparing grade $4 \mathrm{WBC}$ and platelet toxicity, febrile neutropenia and hospitalization rate, RBC and PLT transfusions, and granulocyte colony-stimulating factors (G-CSF) requirement using Fisher's exact test.

Event-free survival (EFS) was calculated from the first day of chemotherapy to recurrence (local or distant), death from all causes, the appearance of secondary tumors, or the last follow-up examination. Overall survival (OS) was calculated from the first day of chemotherapy to death or the last follow-up examination. Survival curves in the two arms and according to the clinical characteristics and chemotherapy-related variables were calculated using the Kaplan-Meier method and compared using the log-rank test.

\section{RESULTS}

The ISG/OS-1 protocol was activated in April 2001 and closed in December 2006. Two hundred forty-six patients who met the eligibility criteria were randomly assigned (Fig 1). Clinical characteristics were well balanced between the two arms (Table 1).

\section{Treatment Compliance}

Case report forms were incomplete for 15 patients (6\%). An early progression was documented in eight patients during the primary phase. For the remaining 223 patients, the ratio between real and planned duration of the chemotherapy treatment was $1.1 \pm 0.3$ for patients in arm $\mathrm{A}$ and $1.24 \pm 0.3$ for patients in $\operatorname{arm} \mathrm{B}(P=.005)$. The median $\mathrm{RD} / \mathrm{I}$ of the protocol was 0.82 . The $\mathrm{RD} / \mathrm{I}$ was $0.92 \pm 0.03$ for patients in arm $\mathrm{A}$ and $0.74 \pm 0.05$ for patients in $\operatorname{arm} \mathrm{B}(P=.02)$.

\section{Toxicity}

Four patients died of causes unrelated to the disease. Two boys, age 11 (arm A) and 14 (arm B) years old, developed an acute, fatal cardiomyopathy. The first patient developed acute cardiomyopathy during an episode of febrile neutropenia after the last CDP cycle. The second patient developed acute cardiomyopathy during the last administration of MTX. Both patients received the cumulative dose of ADM of $420 \mathrm{mg} / \mathrm{m}^{2}$. The echocardiograms performed before ADM administration were in a normal range.

One patient (arm B) died of a disseminated intravascular coagulation that complicated a sepsis possibly related to a surgical infection. One patient (arm B) died of Stevens-Johnson syndrome that arose during an episode of febrile neutropenia.

After high-dose MTX, one patient (arm B) experienced acute renal failure that required a transient dialysis treatment. He is presently alive with mild renal impairment. In seven patients $(\operatorname{arm} \mathrm{A}, \mathrm{n}=$ 3; $\operatorname{arm} \mathrm{B}, \mathrm{n}=4$ ), mild to moderate renal toxicity was reported that 


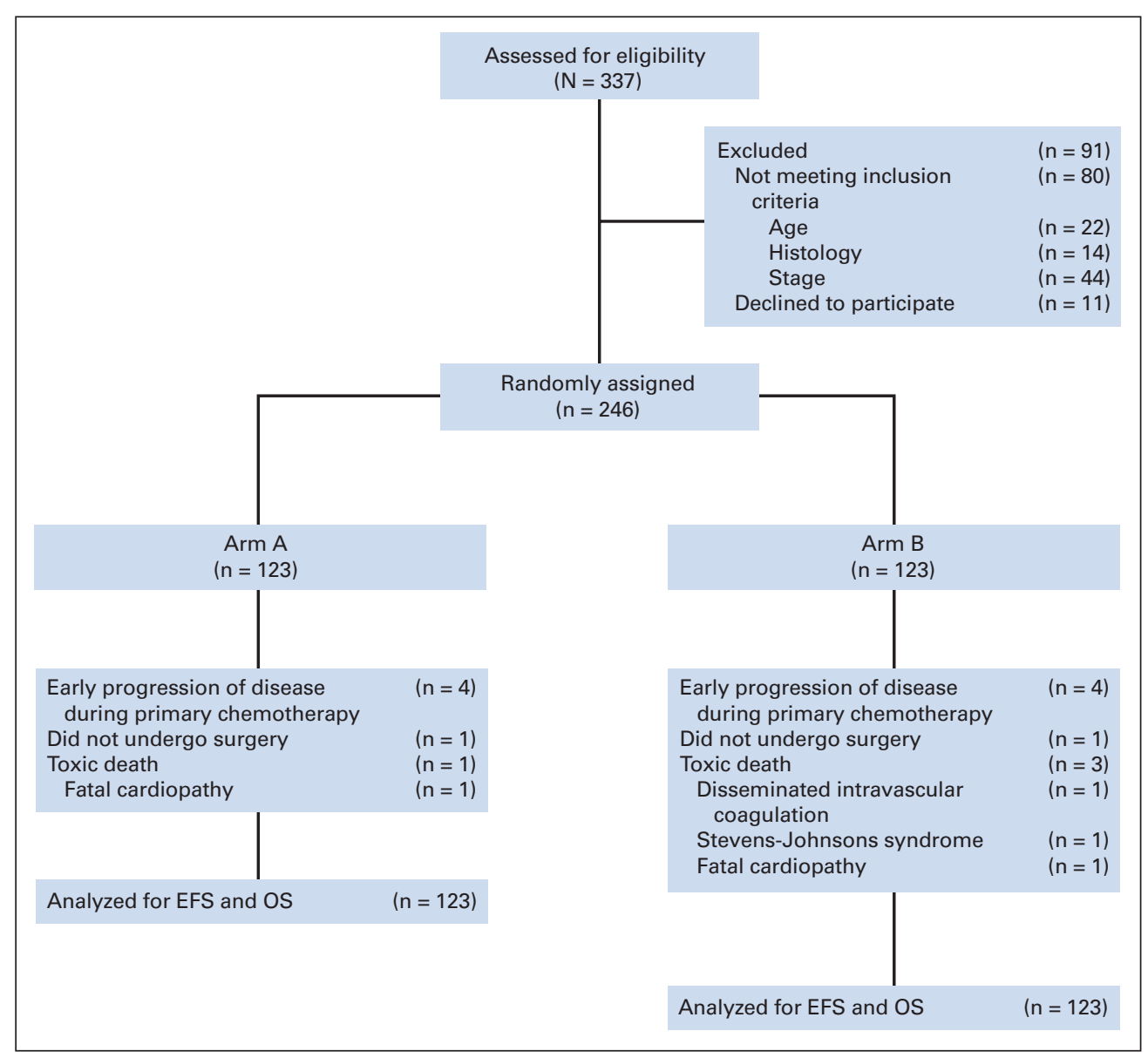

Fig 1. CONSORT diagram. EFS, eventfree survival; OS, overall survival.

caused changes in the planned treatment schedule and no additional administration of nephrotoxic drugs. Four patients are presently alive with renal function in a normal range.

Besides the two deaths already reported, in 11 patients ( $\operatorname{arm~A,}$ $\mathrm{n}=7$; $\operatorname{arm} \mathrm{B}, \mathrm{n}=4$ ), a reduction of ejection fraction of more than $10 \%$ compared with the baseline value was observed. In four patients (arm $\mathrm{A}, \mathrm{n}=3$; $\operatorname{arm} \mathrm{B}, \mathrm{n}=1$ ), the reduction of the ejection fraction was associated with clinical evidence of cardiomyopathy. These four patients are all presently alive and under cardiologic treatment.

In 1,553 cycles of MTX evaluated, the incidence of delayed excretion was $7.6 \%$ in arm $\mathrm{A}$ and $8.2 \%$ in arm B. In only four patients ( $\operatorname{arm} \mathrm{A}, \mathrm{n}=2$; $\operatorname{arm} \mathrm{B}, \mathrm{n}=2$ ), delayed excretion of MTX was associated with persistent renal impairment (in one patient, transient dialysis was required).

Data on hematologic toxicities are listed in Table 2. Patients treated in arm B had a significantly higher incidence of grade 4 leukopenia and thrombocytopenia and more frequently experienced febrile neutropenia requiring more frequent hospitalization, $\mathrm{RBC}$ and or platelet transfusions, and the use of G-CSF. A higher incidence of hematologic toxicity was also observed in arm $B$ when the analysis was restricted to patients in arm A who received all four drugs (data not shown).

\section{Surgery and Histologic Response}

Two patients, one in arm A and one in arm B, did not undergo surgery because of an early systemic progression of the disease. Of the remaining 244 patients, 230 (94\%) underwent conservative surgery, 12 (5\%) underwent amputation, and two (1\%) underwent rotationplasty. Surgical margins, reported in 215 patients, were adequate (radical or wide) in 204 patients (95\%) and marginal in 11 patients (5\%). No differences were observed between the two arms (Appendix Table A2, online only).

Chemotherapy-induced tumor necrosis was rated as good in $45 \%$ of patients ( 110 of 244 patients). The GR rate was $48 \%$ in patients treated in arm $\mathrm{A}$ and $42 \%$ in patients treated in $\operatorname{arm} \mathrm{B}(P=.3)$. The GR rate did not differ according to sex and age but was lower in chondroblastic subtype (Appendix Table A3, online only).

\section{Follow-Up}

A total of 176 patients $(71.5 \%)$ were alive with a median follow-up time of 76 months (range, 31 to 115 months), and the 5 -year probability of OS was 74\% (95\% CI, 68\% to 79\%). Patients in arm A had a 5-year OS of $73 \%$ (95\% CI, 65\% to $81 \%)$, whereas patients treated in arm B had a 5 -year OS of $74 \%$ (95\% CI, 66\% to $82 \% ; P=.6$; Fig 3 ).

One hundred forty-four patients $(58.5 \%)$ were continuously event free. Tumor progression (local or distant) was reported in 95 patients (39\%), four patients died of toxicity, and three patients experienced a second malignant neoplasm (acute lymphoblastic leukemia in two patients and colon cancer in one patient). Local recurrence was reported in 18 patients (in five patients, combined with metastasis). 


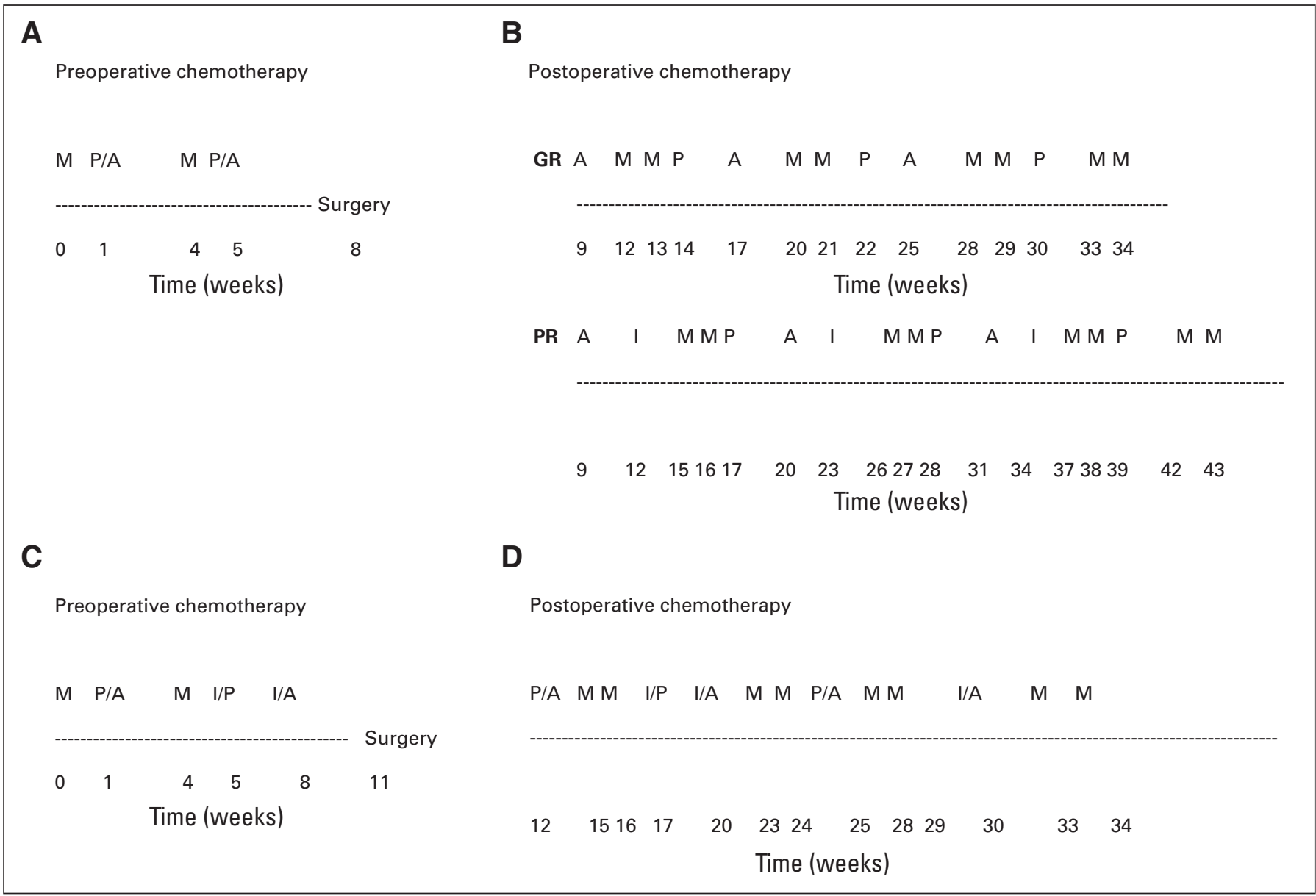

Fig 2. Protocol outline for arm $A(A)$ preoperative chemotherapy and (B) postoperative chemotherapy, using methotrexate $(\mathrm{M}) 12 \mathrm{mg} / \mathrm{m}^{2}$, cisplatin $(\mathrm{P}) 120 \mathrm{mg} / \mathrm{m}^{2}$, doxorubicin (A, adriamycin) $75 \mathrm{mg} / \mathrm{m}^{2}$, and ifosfamide (I) $10 \mathrm{~g} / \mathrm{m}^{2}$, and arm B (C) preoperative chemotherapy and (D) postoperative chemotherapy, using $\mathrm{M} 12 \mathrm{~g} / \mathrm{m}^{2}$, P $120 \mathrm{mg} / \mathrm{m}^{2}, A 70 \mathrm{mg} / \mathrm{m}^{2}$, and I $6 \mathrm{~g} / \mathrm{m}^{2}$. GR, good histologic response; PR, poor histologic response.

The 5-year probability of EFS was $60 \%$ (95\% CI, 53\% to $66 \%)$. Patients in arm A had a 5-year EFS of $64 \%$ (95\% CI, 56\% to $73 \%)$, whereas patients treated in arm B had a 5-year EFS of 55\% (95\% CI, $46 \%$ to $64 \% ; P=.2$; Fig 3 ).

EFS was lower when alkaline phosphatase and lactate dehydrogenase (LDH) were elevated, whereas EFS did not differ according to sex, tumor site, and histologic subtype (Table 3). GR patients had a significantly better probability of EFS compared with PR patients (Table 4). Five-year EFS was 69\% (95\% CI, 60\% to 78\%) for GRs and $52 \%$ (95\% CI, $44 \%$ to $61 \%$ ) for PRs (log-rank $P=.001$ ). Protocol compliance had no impact on EFS (Table 4). Multivariate analyses (via Cox regression) were performed. LDH (high LDH: relative risk, 1.9; 95\% CI, 1.17 to 3.1; $P=.009$ ) and histologic response (GR: relative risk, 0.6 ; $95 \% \mathrm{CI}, 0.38$ to $0.98 ; P=.04$ ) independently influenced EFS.

\section{DISCUSSION}

In patients with high-grade osteosarcoma, chemotherapy was first used in the early 1970s. ${ }^{6}$ Since then, several schemes of multiagent chemotherapy have been assessed, but at present, there is no agreement on a standard chemotherapy regimen. ${ }^{2}$
In our study, we compared two different chemotherapy regimens based on the four active drugs against osteosarcoma, and in particular, we focused on the role of IFO. The drug was used only in case of poor histologic response to the MTX-CDP-ADM regimen ( $\operatorname{arm} \mathrm{A})$ or was added to MTX-CDP-ADM in all patients in the primary phase of chemotherapy (arm B).

The two regimens had a significantly different hematologic toxicity. Patients treated in arm B were more frequently hospitalized because of a higher incidence of neutropenic fever and need of transfusion support. More frequently, patients in arm B required the use of G-CSF. The fact that approximately $50 \%$ of patients treated in arm A did not receive IFO is an important factor but is not the only factor that might explain the different hematologic toxicity. It is important to highlight that, when comparison is made considering only patients in arm A who received IFO, hematologic toxicity still remains significantly higher in arm B. This fact clearly indicates the importance of the administration modality of the drugs and the direct relation between dose-intensity of treatment and hematologic toxicity, which, in our study, was not compensated by a higher use of G-CSF. Toxicity observed in arm B had a direct influence on protocol compliance, which was lower than the compliance reported for arm A. 


\begin{tabular}{|c|c|c|c|c|c|c|c|}
\hline \multirow{2}{*}{$\begin{array}{c}\text { Demographic or Clinical } \\
\text { Characteristic }\end{array}$} & \multicolumn{2}{|c|}{ All Patients } & \multicolumn{2}{|c|}{$\begin{array}{l}\text { Patients in } \\
\text { Arm A }\end{array}$} & \multicolumn{2}{|c|}{$\begin{array}{l}\text { Patients in } \\
\text { Arm B }\end{array}$} & \multirow[b]{2}{*}{$P$} \\
\hline & No. & $\%$ & No. & $\%$ & No. & $\%$ & \\
\hline Total patients & 246 & & 123 & 50 & 123 & 50 & \\
\hline \multicolumn{8}{|l|}{ Age, years } \\
\hline $\begin{array}{l}\text { Median } \\
\text { Range }\end{array}$ & 14 & $4-39$ & 14 & $6-39$ & 14 & $4-34$ & \\
\hline Sex & & & & & & & .8 \\
\hline Male & 146 & 59 & 74 & 60 & 72 & 59 & \\
\hline Female & 100 & 41 & 49 & 40 & 51 & 41 & \\
\hline Site & & & & & & & .3 \\
\hline Femur & 131 & 53 & 62 & 50 & 69 & 56 & \\
\hline Tibia & 60 & 25 & 34 & 28 & 26 & 21 & \\
\hline Humerus & 34 & 14 & 14 & 11 & 20 & 16 & \\
\hline Other & 21 & 8 & 13 & 11 & 8 & 7 & \\
\hline SAP ( $n=209)$ & & & & & & & .3 \\
\hline Normal & 125 & 60 & 65 & 62.5 & 60 & 57 & \\
\hline High & 84 & 40 & 39 & 37.5 & 45 & 43 & \\
\hline $\mathrm{LDH}(\mathrm{n}=198)$ & & & & & & & .2 \\
\hline Normal & 136 & 69 & 72 & 72 & 64 & 65 & \\
\hline High & 62 & 31 & 28 & 28 & 34 & 35 & \\
\hline Histology & & & & & & & .5 \\
\hline Osteoblastic & 157 & 64 & 76 & 62 & 81 & 66 & \\
\hline Chondroblastic & 24 & 10 & 12 & 10 & 12 & 10 & \\
\hline Fibroblastic & 23 & 9 & 11 & 9 & 12 & 10 & \\
\hline Telangiectatic & 23 & 9 & 15 & 12 & 8 & 6 & \\
\hline NOS & 19 & 8 & 9 & 8 & 10 & 8 & \\
\hline
\end{tabular}

A better protocol compliance did not translate to better survival. This is in agreement with the European Osteosarcoma Intergroup and Cooperative Osteosarcoma Study Group experiences regarding the absence of impact on survival of the $\mathrm{RD} / \mathrm{I}$ in patients with nonmetastatic osteosarcoma.,

A similar probability of survival was obtained in the two arms. On the basis of the results of the ISG/OS-1 study, arm A is presently the chemotherapy treatment recommended by the ISG for patients with nonmetastatic osteosarcoma of the extremity outside of clinical trials.

These data are in agreement with those reported in previous studies in which the four drugs had been used. ${ }^{3,9-12}$ A slightly higher
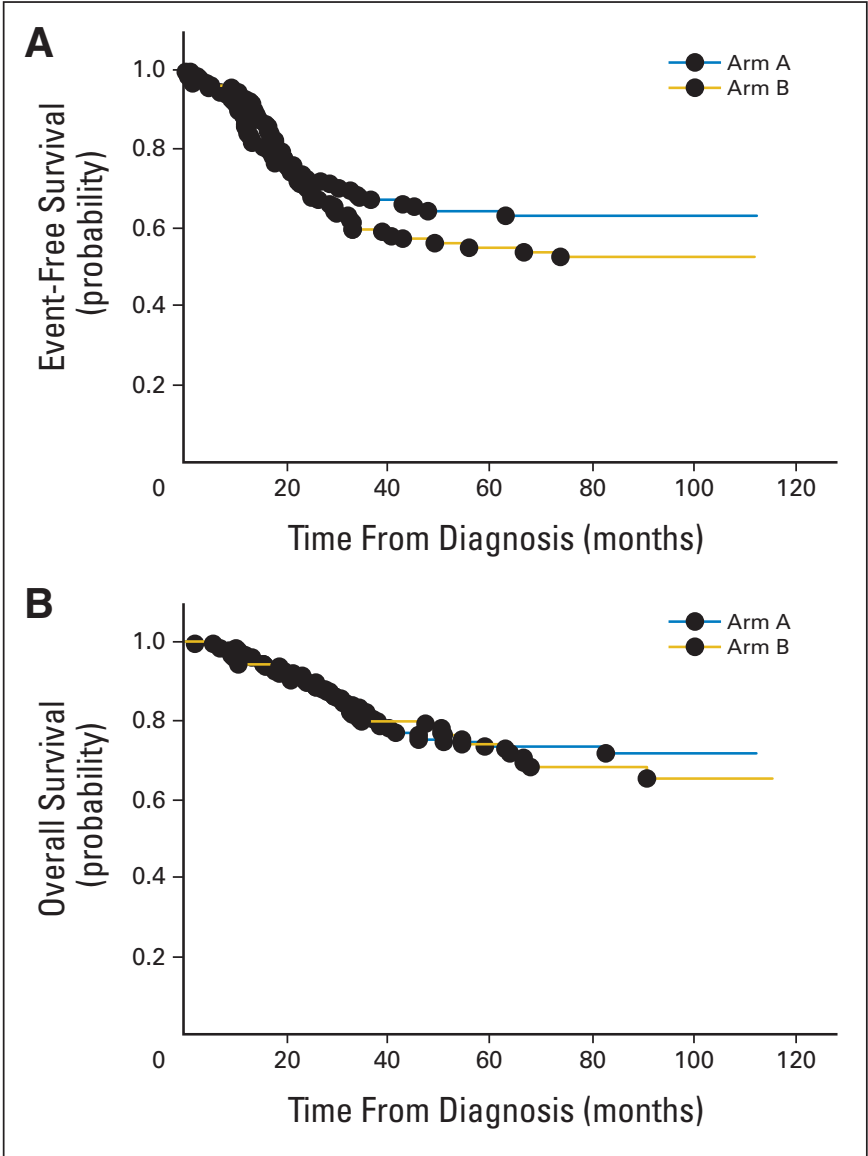

Fig 3. Probability of (A) event-free survival and (B) overall survival by random assignment arm.

probability of survival was reported only in one of the four randomly assigned arms of the last INT-0133 study. ${ }^{13}$ Patients treated with MTX-CDP-ADM plus IFO with the addition of the immunostimulating agent mifamurtide achieved a 6-year probability of survival of $81 \%$. In accordance with previous publications, ${ }^{14,15}$ a good histologic response was predictive of survival in ISG/OS-1.

Our study provides additional information on pathologic response in osteosarcoma because of the possibility of comparing two

\begin{tabular}{|c|c|c|c|c|c|c|c|c|c|c|}
\hline \multirow[b]{3}{*}{ Toxicity } & \multicolumn{4}{|c|}{ Per Cycle } & \multirow[b]{3}{*}{$P$} & \multicolumn{4}{|c|}{ Per Patient } & \multirow[b]{3}{*}{$P$} \\
\hline & \multicolumn{2}{|c|}{$\begin{aligned} & \text { Arm A } \\
(\mathrm{n}= & 865 \mathrm{cycles})\end{aligned}$} & \multicolumn{2}{|c|}{$\begin{array}{c}\text { Arm B } \\
\text { ( } \mathrm{n}=823 \text { cycles })\end{array}$} & & \multicolumn{2}{|c|}{$\begin{array}{c}\text { Arm A } \\
(\mathrm{n}=123 \text { patients })\end{array}$} & \multicolumn{2}{|c|}{$\begin{array}{c}\text { Arm B } \\
(\mathrm{n}=123 \text { patients })\end{array}$} & \\
\hline & No. & $\%$ & No. & $\%$ & & No. & $\%$ & No. & $\%$ & \\
\hline Grade 4 leukopenia & 260 & 30 & 568 & 69 & $<.001$ & 79 & 64 & 117 & 95 & $<.001$ \\
\hline Grade 4 thrombocytopenia & 112 & 13 & 329 & 40 & $<.001$ & 60 & 49 & 103 & 84 & $<.001$ \\
\hline RBC transfusion & 104 & 12 & 280 & 34 & $<.001$ & 43 & 35 & 96 & 78 & $<.001$ \\
\hline PLT transfusion & 52 & 6 & 214 & 26 & $<.001$ & 31 & 25 & 79 & 64 & $<.001$ \\
\hline G-CSF & 554 & 64 & 593 & 72 & $<.002$ & 103 & 84 & 123 & 100 & $<.001$ \\
\hline Neutropenic fever & 138 & 16 & 206 & 25 & $<.002$ & 52 & 42 & 87 & 71 & $<.001$ \\
\hline Hospitalization & 61 & 7 & 165 & 20 & $<.001$ & 54 & 44 & 93 & 76 & $<.001$ \\
\hline
\end{tabular}

Abbreviations: G-CSF, granulocyte colony-stimulating factor; PLT, platelet. 


\begin{tabular}{|c|c|c|c|c|c|c|c|}
\hline \multirow[b]{2}{*}{ Characteristic } & \multirow[b]{2}{*}{ No. of Patients } & \multicolumn{3}{|c|}{ EFS } & \multicolumn{3}{|c|}{ OS } \\
\hline & & 5-Year EFS (\%) & $95 \% \mathrm{Cl}(\%)$ & Log-Rank $P$ & 5 -Year OS (\%) & $95 \% \mathrm{Cl}(\%)$ & Log-Rank $P$ \\
\hline \multicolumn{8}{|l|}{ Sex } \\
\hline Male & 146 & 68 & 59 to 78 & .15 & 71 & 63 to 79 & .16 \\
\hline Female & 100 & 65 & 55 to 74 & & 79 & 70 to 88 & \\
\hline \multicolumn{8}{|l|}{ Age, years } \\
\hline 3-10 & 42 & 78 & 66 to 91 & .07 & 82 & 70 to 94 & .3 \\
\hline $11-17$ & 131 & 58 & 50 to 67 & & 76 & 69 to 84 & \\
\hline$>17$ & 73 & 54 & 42 to 66 & & 65 & 51 to 78 & \\
\hline SAP & 209 & & & & & & \\
\hline High & 84 & 54 & 43 to 65 & .02 & 66 & 57 to 77 & .06 \\
\hline Normal & 125 & 68 & 59 to 76 & & 80 & 73 to 88 & \\
\hline $\mathrm{LDH}$ & 198 & & & & & & \\
\hline High & 62 & 50 & 37 to 63 & .02 & 70 & 58 to 81 & .2 \\
\hline Normal & 136 & 65 & 57 to 73 & & 75 & 68 to 83 & \\
\hline \multicolumn{8}{|l|}{ Histology } \\
\hline Osteoblastic & 157 & 60 & 52 to 68 & .13 & 72 & 65 to 80 & .2 \\
\hline Chondroblastic & 24 & 53 & 31 to 74 & & 71 & 49 to 93 & \\
\hline Fibroblastic & 23 & 71 & 51 to 90 & & 89 & 75 to 100 & \\
\hline Telangiectatic & 23 & 78 & 61 to 95 & & 86 & 72 to 100 & \\
\hline NOS & 19 & 38 & 12 to 63 & & 58 & 31 to 85 & \\
\hline
\end{tabular}

different schemes of primary chemotherapy. No differences were observed between the two arms, and the addition of IFO to MTX-CDPADM did not influence the rate of GR. The two arms were well balanced in terms of factors influencing histologic response such as age and histologic subtypes. ${ }^{16,17}$ The percentage of patients who underwent conservative surgery was $94 \%$; the more intensive four-drug primary treatment did not allow a higher resection rate compared with the MTX-CDP-ADM regimen. These data do not support the use of IFO added to MTX-CDP-ADM in primary chemotherapy of nonmetastatic osteosarcoma of the extremity, at least with the dose and schedule used in our study.

A strategy based on an interval compression in patients with osteosarcoma has been assessed by the European Osteosarcoma Inter- group. ${ }^{18}$ The authors reported that intensification of chemotherapy increased $\mathrm{RD} / \mathrm{I}$ and resulted in an increased response rate, but this did not translate into a better survival compared with the standard chemotherapy arm. These results do not support an interval compression in osteosarcoma.

Results of the present study allow a better understanding of the role of IFO in patients with nonmetastatic high-grade osteosarcoma. Its use in the primary phase added to MTX-CDP-ADM is not recommended, and it is associated with greater hematologic toxicity.

Similar conclusions might be drawn from the results of the INT0133 study. ${ }^{13}$ In the Children's Cancer Group-Pediatric Oncology Group study, patients treated with MTX-CDP-ADM, without the addition of mifamurtide, had a better 6-year EFS (64\%) compared

\begin{tabular}{|c|c|c|c|c|c|c|c|}
\hline \multirow[b]{2}{*}{ Variable } & \multirow[b]{2}{*}{ No. of Patients } & \multicolumn{3}{|c|}{ EFS } & \multicolumn{3}{|c|}{ OS } \\
\hline & & 5-Year EFS (\%) & $95 \% \mathrm{Cl}(\%)$ & Log-Rank $P$ & 5-Year OS (\%) & $95 \% \mathrm{Cl}(\%)$ & Log-Rank $P$ \\
\hline $\mathrm{RD} / \mathrm{l}$ & 223 & & & & & & \\
\hline$<80 \%$ & 110 & 65 & 54 to 75 & .3 & 77 & 67 to 86 & .6 \\
\hline$\geq 80 \%$ & 113 & 58 & 47 to 68 & & 76 & 66 to 85 & \\
\hline Tumor necrosis in all patients & 244 & & & & & & \\
\hline GR & 110 & 69 & 60 to 78 & .001 & 82 & 75 to 90 & .01 \\
\hline $\mathrm{PR}$ & 134 & 52 & 44 to 61 & & 70 & 61 to 78 & \\
\hline \multicolumn{8}{|l|}{ Tumor necrosis in arm A } \\
\hline GR & 59 & 76 & 65 to 88 & .02 & 83 & 72 to 93 & .09 \\
\hline PR & 63 & 56 & 43 to 69 & & 70 & 57 to 82 & \\
\hline \multicolumn{8}{|l|}{ Tumor necrosis in arm B } \\
\hline GR & 51 & 65 & 52 to 78 & .2 & 81 & 70 to 93 & .1 \\
\hline $\mathrm{PR}$ & 71 & 51 & 39 to 64 & & 69 & 58 to 81 & \\
\hline
\end{tabular}


with patients treated with the four-drug combination (MTX-CDPADM-IFO), who had a 6-year EFS of 58\%. The probability of survival at 6 years was $71 \%$ with the three-drug regimen and $70 \%$ with the four-drug regimen. It is interesting to observe that despite the differences in terms of cumulative dose and schedule between the American study and our study, survival results are similar. This fact reinforces the doubt that IFO is needed in first-line chemotherapy in patients with nonmetastatic osteosarcoma of the extremity.

In the interpretation of the results of our study, some limitations have to be considered. The sample size was calculated to detect a difference in survival between the two arms of $15 \%$ or more. In addition, on the basis of previous clinical trials of adjuvant chemotherapy, this percentage may be considered high. This choice was determined by the expected annual enrollment rate of eligible patients by the ISG.

In arm A, IFO was given only in patients with poor response to MTX-CDP-ADM in an attempt to improve their survival. The chemotherapy scheme of arm A was based on a previous protocol (IOR/ OS-2) in which IFO was used only in PRs to primary chemotherapy with MTX-CDP-ADM. In that study, we reported a difference of 11\% in terms of EFS between GRs and PRs who received postoperative IFO (5-year EFS: GR, 67\%; PR, 56\%). ${ }^{19}$

In ISG/OS-1, EFS probability was higher in GR patients compared with PR patients who also received IFO. The study was not designed to explore IFO as salvage chemotherapy in PR patients, and no conclusions in this direction can be drawn. We must keep in mind that a recent SSG report ${ }^{13}$ failed to show an advantage when IFO added to MTX-CDP-ADM was used in PR patients. In the Scandina- vian study, the dose of IFO was similar to that adopted in our study. It is possible that a higher dose or a different schedule might be beneficial in PR patients. A more definitive conclusion could probably come from the results of the EURAMOS- 1 study ${ }^{20}$ where patients who are PRs to MTX-CDP-ADM are randomly assigned to receive or not to receive IFO and etoposide.

The addition of IFO to MTX, CDP, and ADM in the preoperative phase does not improve the rate of patients with a good histologic response and increases hematologic toxicity. IFO should only be considered in patients who have a poor histologic response to MTX, CDP, and ADM.

\section{AUTHORS' DISCLOSURES OF POTENTIAL CONFLICTS OF INTEREST}

The author(s) indicated no potential conflicts of interest.

\section{AUTHOR CONTRIBUTIONS}

Conception and design: Stefano Ferrari, Piero Picci, Gaetano Bacci Provision of study materials or patients: Alessandra Linari Collection and assembly of data: Stefano Ferrari, Pietro Ruggieri, Graziella Cefalo, Angela Tamburini, Rodolfo Capanna, Franca Fagioli, Alessandro Comandone, Rossella Bertulli, Gianni Bisogno, Emanuela Palmerini, Marco Alberghini, Antonina Parafioriti, Piero Picci Data analysis and interpretation: Stefano Ferrari, Graziella Cefalo, Angela Tamburini, Franca Fagioli, Alessandra Linari, Piero Picci Manuscript writing: All authors Final approval of manuscript: All authors

\section{REFERENGES}

1. Marina N, Gebhardt M, Teot L, et al: Biology and therapeutic advances for pediatric osteosarcoma. Oncologist 9:422-441, 2004

2. Hogendoorn PC, ESMO/EUROBONET Working Group, Athanasou N, et al: Bone sarcomas: ESMO Clinical Practice Guidelines for diagnosis, treatment and follow-up. Ann Oncol 21:v204-v213, 2010 (suppl 5)

3. Ferrari S, Smeland S, Mercuri M, et al: Neoadjuvant chemotherapy with high-dose ifosfamide, highdose methotrexate, cisplatin, and doxorubicin for patients with localized osteosarcoma of the extremity: A joint study by the Italian and Scandinavian Sarcoma Groups. J Clin Oncol 23:8845-8852, 2005

4. Enneking WF, Dunham W, Gebhardt MC, et al: A system for the functional evaluation of reconstructive procedures after surgical treatment of tumors of the musculoskeletal system. Clin Orthop Relat Res 286:241-245, 1993

5. Picci P, Bacci G, Campanacci M, et al: Histologic evaluation of necrosis induced by chemotherapy: Regional mapping of viable and nonviable tissue. Cancer 56:1515-1521, 1985

6. Jaffe N, Paed D, Farber S, et al: Favorable response of metastatic osteogenic sarcoma to pulse high-dose methotrexate with citrovorum rescue and radiation therapy. Cancer 31:1367-1373, 1973

7. Lewis IJ, Weeden S, Machin D, et al: Received dose and dose-intensity of chemotherapy and outcome in nonmetastatic extremity osteosarcoma: European Osteosarcoma Intergroup. J Clin Oncol 18:4028-4037, 2000
8. Eselgrim M, Grunert $H$, Kühne $T$, et al: Dose intensity of chemotherapy for osteosarcoma and outcome in the Cooperative Osteosarcoma Study Group (COSS) trials. Pediatr Blood Cancer 47:42-50, 2006

9. Fuchs N, Bielack SS, Epler D, et al: Long-term results of the co-operative German-Austrian-Swiss Osteosarcoma Study Group's protocol COSS-86 of intensive multidrug chemotherapy and surgery for osteosarcoma of the limbs. Ann Oncol 9:893-899, 1998

10. Le Deley MC, Guinebretière JM, Gentet JC, et al: SFOP OS94: A randomised trial comparing preoperative high-dose methotrexate plus doxorubicin to high-dose methotrexate plus etoposide and ifosfamide in osteosarcoma patients. Eur J Cancer 43:752761, 2007

11. Smeland $S$, Müller $C$, Alvegard $T A$, et al: Scandinavian Sarcoma Group Osteosarcoma Study SSG VIII: Prognostic factors for outcome and the role of replacement salvage chemotherapy for poor histological responders. Eur J Cancer 39:488-494, 2003

12. Smeland $S$, Bruland $O S$, Hjorth $L$, et al: Results of the Scandinavian Sarcoma Group XIV protocol for classical osteosarcoma: 63 patients with a minimum follow-up of 4 years. Acta Orthop 82:211-216, 2011

13. Meyers PA, Schwartz CL, Krailo MD, et al: Osteosarcoma: The addition of muramyl tripeptide to chemotherapy improves overall survival-A report from the Children's Oncology Group. J Clin Oncol 26:633-638, 2008

14. Bielack SS, Kempf-Bielack B, Delling G, et al: Prognostic factors in high-grade osteosarcoma of the extremities or trunk: An analysis of 1,702 patients treated on neoadjuvant cooperative osteosarcoma study group protocols. J Clin Oncol 20:776-790, 2002
15. Bacci G, Longhi A, Versari $M$, et al: Prognostic factors for osteosarcoma of the extremity treated with neoadjuvant chemotherapy: 15-year experience in 789 patients treated at a single institution. Cancer 106:1154-1161, 2006

16. Hauben El, Weeden S, Pringle J, et al: Does the histological subtype of high-grade central osteosarcoma influence the response to treatment with chemotherapy and does it affect overall survival? A study on 570 patients of two consecutive trials of the European Osteosarcoma Intergroup. Eur J Cancer 38:1218-1225, 2002

17. Bacci G, Bertoni $F$, Longhi $A$, et al: Neoadjuvant chemotherapy for high-grade central osteosarcoma of the extremity: Histologic response to preoperative chemotherapy correlates with histologic subtype of the tumor. Cancer 97:3068-3075, 2003

18. Lewis IJ, Nooij MA, Whelan J, et al: Improvement in histologic response but not survival in osteosarcoma patients treated with intensified chemotherapy: A randomized phase III trial of the European Osteosarcoma Intergroup. J Natl Cancer Inst 99:112-128, 2007

19. Bacci G, Ferrari S, Bertoni F, et al: Long-term outcome for patients with nonmetastatic osteosarcoma of the extremity treated at the Istituto Ortopedico Rizzoli according to the Istituto Ortopedico Rizzoli/Osteosarcoma-2 Protocol: An updated report. J Clin Oncol 18:4016-4027, 2000

20. Marina N, Bielack S, Whelan J, et al: International collaboration is feasible in trials for rare conditions: The EURAMOS experience. Cancer Treat Res 152:339-353, 2010 